\title{
Veränderliche Sterne 1883, beobachtet zu Athen.
}

Ohne an der Form der Resultate und an dem provisorischen Charakter derselben etwas zu ändern, gebe ich die Maxima und Minima des Lichtes in derselben Weise, wie ich sie alljährlich seit 1855 mitgetheilt habe; es sind die Ergebnisse des 42 sten Jahrganges meiner Beobachtungen über veränderliche Sterne. Alle Vergleichungen erhielt ich zu Athen; die telescopischen am Sucher oder am 5 fuiss. Refractor von Reinfelder. Die Zeit ist mittlere Ortszeit, $d$ die Dauer, $p$ das Gewicht der Beobachtung, $h$ die Helligkeit des Sternes.

\section{Algol 1883. Minima.}

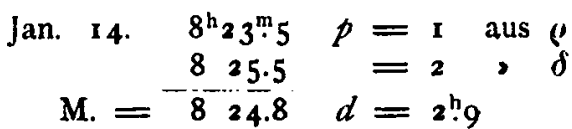

22 Beob., Luft ungünstig, dunstig bei Mondschein.

Febr. 6. $6^{\mathrm{h}} 54^{\mathrm{n}} \cdot 0 \mathrm{o}=\mathrm{I}$ aus $\rho$

$$
\begin{aligned}
655.5 & =2 \\
6 & =1
\end{aligned}{ }^{6} .08
$$

40 Beob. Das Min. fiel auf das Ende des Tages.

Aug. 11. $15^{\mathrm{h}} 30^{\mathrm{m}} \quad p=0.5$ aus $\varphi$

$$
\text { M. }=\frac{1535}{1533} \quad d=1, \quad \delta
$$

I 8 Beob. Min. der Dämmerung zu nahe.

Aug. 14. $12^{\mathrm{h}} 37^{\mathrm{m}} \cdot 5 \quad p=1$ aus

$$
\mathrm{M} .=\frac{1237.0}{1237.2} \quad d=2,2^{\mathrm{h}}, \quad \delta
$$

3 I Beob. Sehr klar, erst seit I ${ }^{\text {h }} 9$ zahlreiche Beob. vorher grosse Lücke.

Sept. 3. $12^{\text {h }} 40^{\text {in }}$ war das Min. schon vorüber.

Sept. 6. II $I^{\mathrm{h}} 23^{\mathrm{m}} \cdot 5 \quad p=I$ aus $\varrho$

$$
\mathrm{M} .=-\frac{1}{\mathrm{II}}-\frac{22.5}{22.8} \quad d=4.5
$$

56 Beob. Sehr klar, anfangs stand Algol noch sehr tief. Beob. vollständig.

$$
\text { Sept. 29. } \begin{array}{rl}
9^{\mathrm{h}} 43^{\mathrm{m}} \cdot 0 & p=1 \\
940.0 & =2 \\
9 & =2 \mathrm{I} .0 \quad d=4^{\mathrm{h}} \cdot 3
\end{array}
$$

$3^{8}$ Beob. Meist klar; $9: 4$ bis $10 ! \mathrm{I}$ fehlen die Beob.

$$
\begin{aligned}
& \text { Oct. I } 9 . \text { I } 1^{\mathrm{h}} 7^{\mathrm{m}} \circ \mathrm{O}=1 \text { aus } \varphi
\end{aligned}
$$

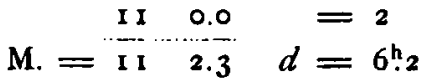

18 Beob. Sehr klar, Mond stark seit $9^{\text {h }}$. Nur gelegentliche Beob.

$$
\begin{aligned}
& \text { Oct. 22. } 8^{\text {h }} 3^{\text {th }} \circ p=1 \text { aus } \rho \\
& \begin{aligned}
\text { M. }=\frac{8}{8} \frac{5.0}{8} \quad 4.3 & =2 \\
& =3^{\mathrm{h}} \cdot 3
\end{aligned}
\end{aligned}
$$

$3^{2}$ Beob. Meist klar bis $7^{\mathrm{h}} \cdot 5$, später dunstig.

$$
\begin{aligned}
& \text { Nov, I I. } 9^{\mathrm{h}} 3^{8 \mathrm{~m}} \circ \mathrm{o} \quad p=1 \text { aus } \rho \\
& \begin{aligned}
\text { M. }=\frac{947.0}{944.0} \quad d=2 & =4^{\mathrm{h}} .8
\end{aligned}
\end{aligned}
$$

28 Beob. Nahe Vollmond; wegen Wolken fehlen Beob. von $7^{\text {h }}$ bis $9^{\text {h }}$.

$$
\text { Dec. 4. } \begin{array}{rl}
8^{\mathrm{h}} 15^{\mathrm{m}} \cdot \mathrm{o} & p=1 \text { aus } \rho \\
818.0 & =2 \\
\mathrm{M} .=\frac{817.0}{8}=3^{\mathrm{h}} \cdot 9
\end{array}
$$

48 Beob. Meist klar, doch feinster Dunst; Mond bis 8. 5 . Um die Zeit des Min. fehlen durch 20 Minuten die Beob.

$$
\text { Dec. 7. } 5^{\mathrm{h}} 4^{8^{\mathrm{m}}} \quad p_{d}=0.5 \text { aus } \delta
$$

r 6 Beob. Min. am Ende des Tages, daher ganz unsicher.

$$
\text { 2. } \varepsilon \text { Aurigae. }
$$

Die sehr geringen Aenderungen des Lichtes (1 85 Beob.) machen eine nähere Untersuchuug überfluissig.

$$
\text { 3. C Cassiopeae. }
$$

Zahlreiche Vergleichungen mit $\beta$ und $\gamma$ zeigen nur jene kleinen Aenderungen, die sehr wesentlich von der Lage der Verbindungslinie beider Augen gegen die Verbindungslinie der verglichenen Sterne abhängen, so dass eine specielle Untersuchung doch kein entscheidendes Resultat ergeben würde.

\section{Mira Ceti $188_{3}$.}

Die Beob. für das kleinste Licht umfassen die Zeit 1882 Winter bis 1883 März; es fehlen aber Vergleichungen Dec. 1 2-26. Alle geschahen am 5 f. Refr. Sie geben die letzte Abnahme rascher als die erste Zunahme. Ich setze : Min. I882 Dec. I6 recht sicher. Der Stern ward dem nachfolgenden Begleiter an Helligkeit gleich, oder blieb unmerklich heller, weniger als $1 / 2$ Stufe. Das Maximum im Sommer 1883 konnte in der Frühdämmerung nicht gesehen werden. Juli 25, 27, 29, 3I war Mira, tief in der Dämmerung, dem freien Auge nicht sichtbar. Ich schätzte ihn am Sucher Juli $27=6^{\mathrm{m}} \cdot 2$, Juli $29=6^{\mathrm{m}} \cdot 7$, Aug. $10=6 \mathrm{~m} \cdot 8$. Seit Aug. 3 I beob. ich ihn am Refr. für das Minimum. Die Beob. von Oct. 1883 bis Anfang. Jan. 1884 ergaben: $M$ in. 1883 Nov. 28 gut. Helle sehr wenig geringer als die des Nachbarsternes. Periode seit dem letzten Min. $=347 \pm 5$ Tage.

\section{R Hydrae $188_{3}$.}

$\begin{array}{rrlr}\text { Febr. } 3 & \mathrm{R}=9.2 & \text { April } 15 & \mathrm{R}=6.5 \\ 28 & 7.8 & \text { Mai } 25 & 5.2 \\ \text { März } 7 & 7.5 & \text { Juni } 28 & 6.8 \\ \text { I } 8 & 7.2 & \text { Juli } 25 & 7.0 \\ 3 \text { I } & 7.0 & & \end{array}$

Um die Zeit des grössten Lichtes und bald nachher war starker Mondschein, der nicht zuliess, R ohne Fernrohr 
zu sehen; daher beob. ich ihn zu jener Zeit mit dem Sucher, ohne indessen diesen Vergleichungen Einfluss auf die Curve zu gestatten. Ich finde:

Max. I883 Mai 15.0 gut. $h=55^{\mathrm{m}}$. Die Beob. am Sucher ergaben Mai 18 , weniger sicher.

$$
\text { 6. } \delta \text { Librae } 1883 \text {. }
$$

M in im a Mai 29. $11^{\mathrm{h}} 15^{\mathrm{m}}$, von 8.6 bis $13^{\mathrm{h}} \cdot 5$, wenig beob. Juni 5. Io $50,8.7$ 1 2.6, gute Beob.

Am 1 2. und 26. Juni ward das Min. am Sucher zu bestimmen versucht, doch ohne Erfolg.

Dem Min. nahe sah ich $\delta$

$$
\begin{aligned}
& \text { Juli ro von } 9.4 \text { bis } 10^{h: 6} \\
& 24>9.1 \times 9.8 \\
& 31>8.8>9.6
\end{aligned}
$$

dann: Aug. $218^{\text {h }}$ x und Sept. $47^{\text {h }} \cdot 9$.

$$
\text { 7. } y \text { Aquilae } 1883 \text {. }
$$

Maxima.

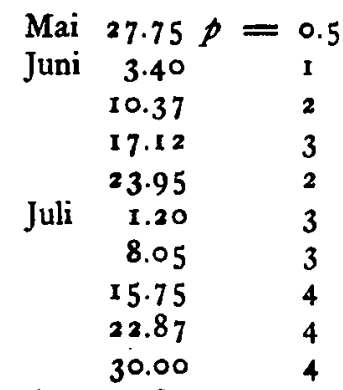

Aug. 5.80

13.57

20.75

27.87

Sept. 3.75

10.90

18.30

26.82

Oct. $\quad 2.77$

9.33

17.20

24.80

31.50

Noy. 7.90

$\mathrm{I} \cdot 5.85$

22.00

29.45

Dec. 6.65
Minima.

$$
\begin{aligned}
& \text { Mai } 30.90 p=1 \\
& \text { Juni } 7.12 \quad 0.5 \\
& 14.02 \quad 3 \\
& \text {, } 20.87 \text { 2 } \\
& 28.67 \quad 3 \\
& \text { Juli } 6.07 \quad 3 \\
& 13.05 \\
& 20.37 \\
& 27.25 \\
& \text { I } 5.25 \\
& 22.85 \\
& 3020 \\
& 28.85
\end{aligned}
$$

\begin{tabular}{|c|c|c|}
\hline $\begin{array}{l}\text { Mai } \\
\text { Juni }\end{array}$ & $\begin{array}{r}20.00 \\
1.98\end{array}$ & $F=$ \\
\hline & $\begin{array}{l}15.90 \\
28.95\end{array}$ & \\
\hline Juli & 12.00 & \\
\hline & 24.45 & \\
\hline Aug. & $5.5^{\circ}$ & \\
\hline & 19.05 & \\
\hline Sept. & I.30 & \\
\hline & 14.35 & \\
\hline & 27.50 & \\
\hline
\end{tabular}

Dec. 3.70

$$
11
$$

8. dCephei 1883 .

\section{Maxima.}

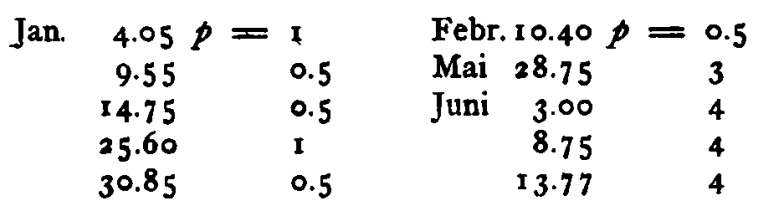

Maxima.

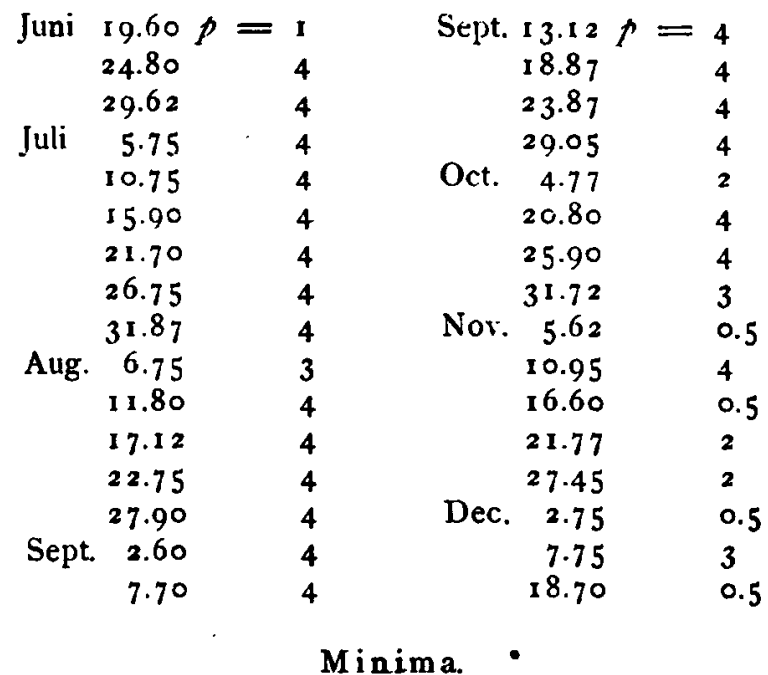

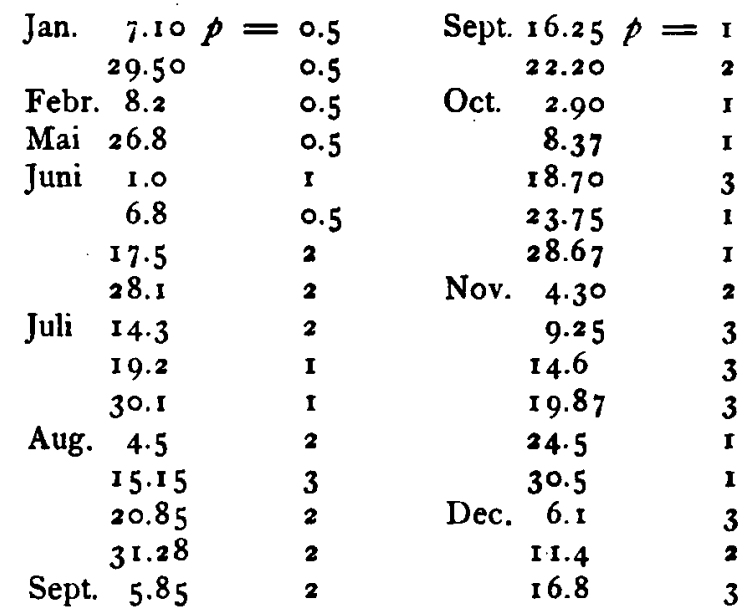

Im Allgemeinen lassen sich aus meinen Beob. keine sicheren Minima ableiten. Doch habe ich einige Angaben hergesetzt, in Fällen, da die Curve weniger flach, und einigermaassen geeignet erschien, ein Minimum versuchsweise zu bestimmen. Alle Athener Beob. von Febr. bis Ende Mai, besonders im März und April, sind wegen der tiefen Lage des Sternes am Nord-Horizonte ganz unsicher, und verdienen keine Berücksichtigung.

$$
\text { 9. } \beta \text { Lyrae } \mathrm{I}_{883} \text {. }
$$

Hauptminima.

\begin{tabular}{lrr} 
Mai & \multicolumn{1}{c}{ I } \\
27.72 & $p=0$ \\
Juni & 9.20 & 3 \\
& 22.17 & 3 \\
Juli & 4.85 & 4 \\
& 17.95 & 4 \\
& 30.67 & 4 \\
Aug. 12.87 & 4 \\
& 25.65 & 4 \\
Sept. 7.80 & 4 \\
& 20.70 & 2
\end{tabular}

Nebenminima. 


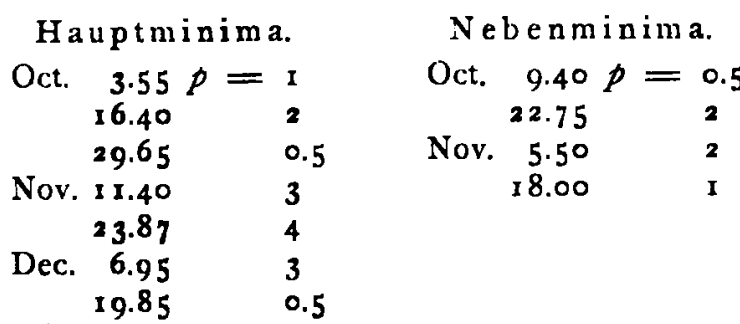

Kein Nebenminimum ist wirklich sicher bestimmt; es ward einmal nahe so lichtschwach wie das Hauptminimum.

$$
\text { 10. } \eta \text { Geminorum } 188_{3} \text {. }
$$

Die Beob. in 1883 zeigen, dass die ehemalige Periode von $225^{\mathrm{d}}$ nicht mehr stattfindet. Iie beiden genügend sicher bestimmten Minima fallen auf Jan. 29 und Nov. 27. Das hypothetische Juni 8 konnte wegen der damaligen Lage des Sternes nicht gesehen werden. Zwei Maxima werden durch die Curve auf April 14 und Aug. 27 verlegt. Die Periode aus dem $M \dot{a} x$. ist $=15^{I^{d}}$, die aus dem $M$ in. $=135^{\mathrm{d}}$, eine genügende Uebereinstimmung, da der $\mathrm{w} . \mathrm{F}$. jeder Epoche mindestens $\pm 10^{\alpha}$ beträgt.

$$
\text { 11. Geminorum } 1883 \text {. }
$$

Sehr wenig befriedigend sind die Resultate dieses Jahres, obgleich sich der Stern zu jeder Zeit sehr vortheilhaft mit seinen Nachbarn $\delta$ und $\lambda$ vergleichen lässt. Die oft ungünstige Luft erklärt nicht die Anomalien der Curve und die geringe Sicherheit der Epochen. Ich weiss aber seit mehr als 30 Jahren, dass dieser Stern (wie auch andere) plötzliche Unregelmässigkeiten im Lichtwechsel zeigt. - In der Uebersicht der Max. und Min. findet man noch einige Angaben für Dec. I882, die in meinem letzten Berichte übersehen wurden.

Die ganz abweichenden Angaben bezeichne ich durch *.

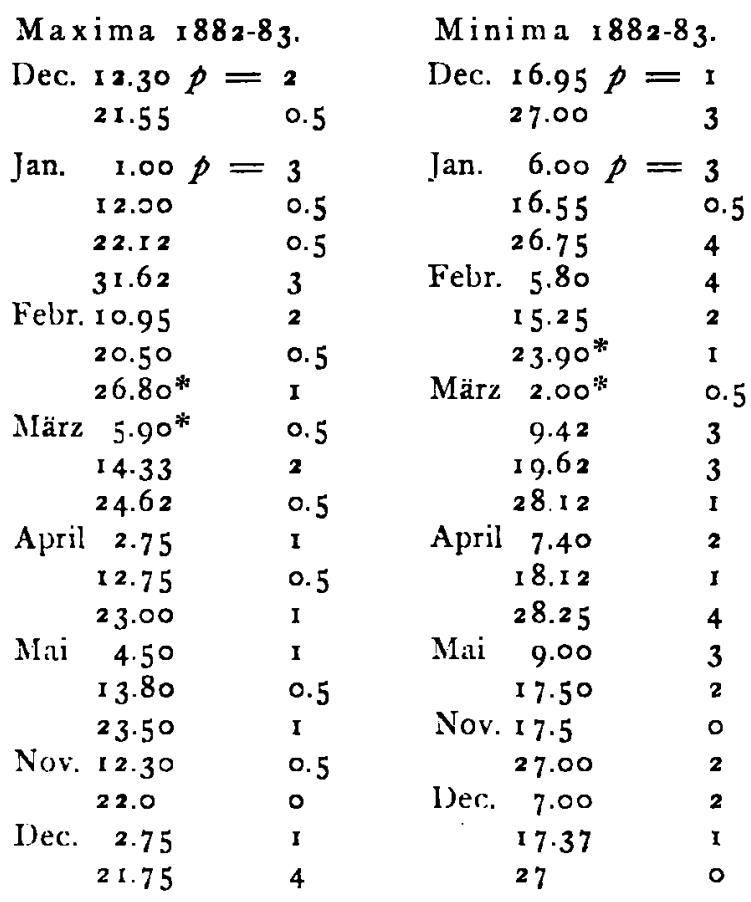

\section{2. a Herculis 1883 .}

Aus vielen Beob.von Jan. bis Dec. lässt sich Folgendes gut bestimmen:

\author{
Max. Mai 26 gut. \\ Min. Juli 19 , \\ Max. Sept. 7 ziemlich.
}

Periode $=54$ und 60 Tage.

$$
\text { 13. g Herculis. } 1883 \text {. }
$$

Die Aenderungen des Lichtes waren häufig und stark. In der Jahrescurve haben die Scheitel der Maxima nach und nach eine tiefere Lage, oder, die maximale Helligkeit nahm langsam ab.

$$
\begin{array}{lll}
\multicolumn{2}{c}{\text { M a x ima. }} & \multicolumn{2}{c}{\text { Minima. }} \\
\text { April } \text { I } 5 \text { gut. } & \text { Febr. } 24 \text { unsicher. } \\
\text { Juli } 17 \text { gut. } & \text { Juni } 8 \text { gut. } \\
\text { Oct. } 7 \text { ziemlich. } & \text { Sept. } 9 \text { gut. } \\
& & \text { Dec. } 8 \text { unsicher. }
\end{array}
$$

Von Febr. 25 abgesehen, erhält man Perioden von 93, 82, 83, 90 Tagen. Es folgt aber auf jedes Maximum ein secundäres Minimum, bevor das wahre Minimum eintritt.

$$
\text { 14. } \alpha \text { Orionis } \mathbf{1 8 8 3} \text {. }
$$

Allzu schwache Aenderungen des Lichtes lassen die nähere Bestimmung erfolglos erscheinen.

\section{5. $\beta$ Pegasi 1883 .}

In Januar und Februar, da der Stern tiefe Lage hatte, führten die Beob.zu keinem Resultate. Später liessen sich wohl die Extreme der Curve darstellen, aber es zeigte sich, dass die Maxima zumeist mit der hellsten Mondphase zusammenfielen:

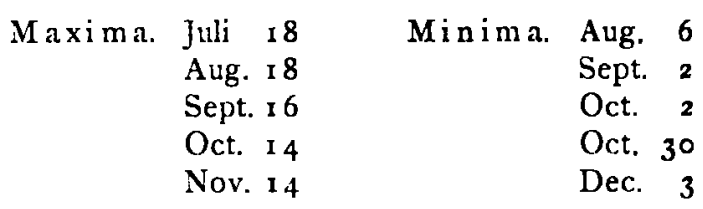

Im Mittel folgt daraus Periode $=29.7$ 'Tage.

$$
\text { 16. } \rho \text { Persei } 1883 \text {. }
$$

Der Stern war nur schwach veränderlich, der Entwurf der Curve ist für jetzt ohne Interesse.

\section{7. u Hercul is $\mathbf{r} 883$.}

Für diesen Stern unterlasse ich auch diesmal die nähere Untersuchung, da sie nach dem vormals Mitgetheilten nichts von Bedeutung hinzufigen würde; u zeigte 1883 ebenfalls die von mir nachgewiesenen seltenen Erscheinungen. Nach je 37-40 Tagen war der Stern vorwiegend lichtschwach, und man sah dann in kurzen Perioden die sehr grossen Variationen, die den Maximalzeiten fehlen. Solche Zeiten waren um Mai ro, Juni 15, Juli 24, Aug. 2 I etc. Vom Oct. an habe ich sie nicht beinerkt. 


\section{R Andromedae 1883 .}

Nach dem Max. im Oct. 1882 nahm der Stern langsam ab, und war 1883 Jan. $28=9^{\mathrm{m}}$. Juli 15 , Sept. 9 war er am Sucher nicht kenntlich, Sept. 2 I $=9^{\prime \prime} \cdot 6$, Oct. 18 $=8^{\mathrm{m}} \cdot 2$, Nov. $3=7^{\mathrm{m}} \cdot 5$. Die Vergleichungen Nov. 4 bis Jan. 17 (1884) zeigen Anfangs eine sebr rasche Zunahme. Nov. $3^{\circ}$ bis Jan. 4 war die Aenderung schwach. Ungeachtet der beschwerlichen Zenithlage ist die Zeit des grössten Lichtes ziemlich gut bestimnt.

M ax. 1883 Dec. $15 \mathrm{~h}=7^{\mathrm{m}}$. Seit Mitte Jan. 1884 war die Abnahme entschieden.

\section{R Aquarii" $188_{3}$.}

Nach dem Max. im Winter 1882 wurden die Beob. bis 1883 Jan. Io fortgesetzt. Juli 27 bis Aug. 27 war $R$ am Sucher nicht sichtbar. Aug. 29 erste Spur, dann $R$ im Sept. schwach, sehr langsam zunehmend von $9: 7$ bis $9^{\mathrm{m}}$, Nov. $15=8 \mathrm{~m} 8$. Die regelmässigen Beob. begannen Oct. 19 . 1884 Jan. 17 schien $R$ schon abgenommen zu haben; die Ermittelung des grössten Lichtes erfolgt erst später.

$$
\text { 2o. R'Arietis } 1883 \text {. }
$$

Aus Vergleichungen von $188_{2}$ Sept. bis $188_{3}$ Ende Febr. folgt: $\mathrm{Max} .1883$ Jan. $15, \mathrm{~h}=8^{\mathrm{m}}$ ziemlich sicher. Als später die Beob. am Morgenhimmel begannen, fand ich Juli $27 \quad R=7^{\mathrm{m}} \cdot 7$, aber schon abnehmend, so dass er Oct. 5 am Sucher nicht mehr gesehen ward. Am Refr. schien $\mathrm{R}$ vielleicht $=\mathrm{I}^{\mathrm{m}}{ }^{\mathrm{m}}$. Bis Mitte Jan. 1884 blieb der Stern für den Sucher unkenntlich; aber Jan. 20 sah ich ihn $=9^{m}$.

\section{T Aquarii 1883 .}

Jie Beob. für das Max. begannen Anfang Dec. I882, aber schon 1883 Jan. II konnte ' $T$ ' in der Dämmerung nicht mehr gesehen werden (am Sucher). Genähert giebt die Curve: $M$ ax. 1883 Jan. 5, $\mathrm{h}=7^{\mathrm{m}} \cdot 5$. Spätere sehr zahlreiche Beob. ergaben: Max. I883 Juli $31, \quad h=7^{\mathrm{m}} \cdot 8$ nach 3 Sternen. Die Zunahme erfolgte sehr rasch, dann längeres Verweilen im Maximum, und schnellere Abnahme seit Aug. 16.

\section{R Aurigae $188_{3}$.}

Max. 1883 Jan. 30.0, $\mathrm{h}=7.8$ gut; die Zunahme schneller als die Abnahme, welche letztere bis Mai 26 anhielt, als der Stern Abends kaum noch in der Dämmerung erkannt ward. Seit Juli 27 ward er Morgens beobachtet. Nach langsamer Abnahme erfolgte ein $M$ in. 1883 Nov. 3, $\mathrm{h}=9{ }^{\mathrm{m}} \cdot \mathrm{2}$, dann raschere Zunahme.

\section{R Bootis 1883}

1883 Febr. $3 \mathrm{R}=9^{\mathrm{m}} .6$; seit Febr. 28 unsichtbar am Sucher. Die späteren Beob. reichen von Mai 11 bis Sept. 5 (Sucher); von diesen benutzte ich 2 Reihen, Juni ro bis Aug. 3o, um das grösste Licht zu bestimmen. Dje Curven ergaben: M ax. 1883 Juli 5.1, $h=6^{m} \cdot 5$. Die Zunahme vielleicht ein wenig rascher als die Abnahme.

$\Delta \mathrm{m}$ Refr. fand ich Mai I $\mathrm{R}=\mathrm{I}^{\mathrm{m}}$, Mai $9=9^{\mathrm{m}} \cdot \mathrm{q}$. Das spätere Mininum im Herbste versuchte ich am Refr. zu bestimmen, und zwar seit Aug. 26. Aber Oct. 26 war die Lage schon so niedrig, dass $R$ unsichtbar blieb. Oct. 2 $\mathrm{R}=$ I0 $^{\mathrm{m}} 5$. Oct. $6 \mathrm{R}=1 \mathrm{I}^{\mathrm{m}}$. Oct. $19 \mathrm{R}=1 \mathrm{I}^{\mathrm{m}} \cdot 5$, sehr tief stehend. Dec. $22 \mathrm{R}$ am Sucher nicht sichtbar.

\section{S Bootis 1883 .}

1882 Oct. 29 bis 1883 April 3 ward $S$ am Sucher nicht erkannt. April I $\mathrm{S}=9 \mathrm{~m}_{5}$. Aus 2 Curven hat sich ergeben: Max. 1883 Mai 22.2, $h=8^{\mathrm{m}}$. Zunahme etwas rascher als Abnabme. Aug. 24 ward $R$ am Sucher unsichtbar. Dec. 22 war er $=9.2$.

\section{T Cephei 1883 .}

Max. 1883 Jan. $27, h=7^{m}$ nach vielen Beob. am Sucher. Vielleicht war es sclion Jan. 23, aber den Beob. bei starken Mondscheine gestatte ich nur geringen Einfluss auf die Figur der Curve. Die Zunahme war etwas rascher, als die Abnahme. Mai $9{ }^{\prime} \mathrm{T}=8^{\mathrm{m}}$. . Mai $20=9^{\mathrm{m}}$. Aug. 4 $\mathrm{T}$ am Sucher unsichtbar. Aug. $25 \mathrm{I}=10^{\mathrm{m} .2} \mathrm{am}$ Refr. Sept. 30 begannen auf's Neue die Beob. am Sucher. Indessen werde ich das Max. erst später bestimmen können, da sich Mitte Jan. 1884 noch keine Abnahme zeigte.

$$
\text { 26. U Cephei } 188_{3} \text {. }
$$

Nur eine vollständige Beob. des kleinsten Lichtes gelang am Sucher, Nov. $45^{\mathrm{b}} \cdot 9$ bis $12^{\mathrm{b}} \mathrm{x}$. Die Abnahme, das lange Verweilen in kleinsten Lichte, die Zunahme erfolgte genau so, wie es früher gesehen ward. 2 Curven ergeben: $M$ in. 1883 Nov. $49^{\text {h }} 6^{\mathrm{m}}$ gut. Ausserdem erhielt ich Oct. 20, 25, Nov. 4, I 4, 29 noch gute Angaben für die Zeiten, wann $U$ mit seinen 2 Nachbarn dieselbe Helligkeit hatte.

\section{R Cephei $\mathbf{8 8 3}$}

Dieser Stern (zwischen $\delta$ und $\alpha$ Ursae min.) ward regelmässig beobachtet. Ich werde später Alles, was ich aus den Vergleichungen seit einer Reihe von Jahren gefunden habe, mittheilen. Schönfeld's Angabe für die Periode wird sich bestätigen.

\section{R Coronae 1883 .}

Die bedeutende Helle des Sternes, die schon so lange gedauert hatte, blieb auch 1883 bis Mitte Mai nahe ungeändert. Seit Mai I $_{7}$ hat das Licht stark abgenommen und $R$ blieb am Sucher unsichtbar von Juni 10 bis Sept. 5, als er sich wieder $=9^{\mathrm{m}} \cdot 5$ wahrnehmen liess. Sept. 28 hatte er schon $7^{\mathrm{m}} \cdot 5$. Obgleich die Beob. am Refr. wegen der Lichtschwäche des Sternes nicht ausreichen, ist doch anzunehmen, dass um die Mitte des August ein $M i n .=12^{m}$ eingetreten sei, dem vielleicht Anfangs August ein secundäres lichtschwaches Maximum voranging. Der Verlauf der Aenderungen war ungefähr folgender:

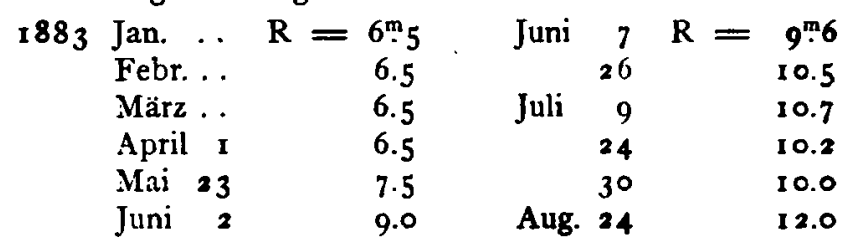




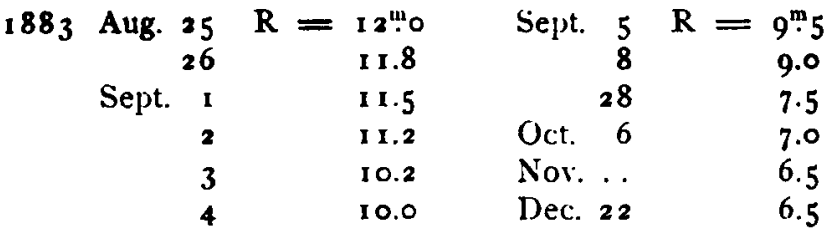

29. S Coronae 1883 .

1883 März $26 \mathrm{~S}=9^{\mathrm{m} .2}$. Die Beob. am Sucher bis Ende Juli haben nach 3 Curven ergeben: Max. I $88_{3}$ Mai I.5, $\mathrm{h}=7^{\mathrm{m}} \cdot$. Aug. 8 war $\mathrm{S}=9^{\mathrm{m}} \cdot 2$. Zunahme rasch, die Abnahme bis Anfang Juni langsam, dann schneller.

\section{T Coronae 1883.}

Es ist die Nova von 1866 . Auch jetzt noch ist der für den Sucher schon zu lichtschwache Stern veränderlich, und war besonders im Juni kaum sichtbar; im August war wohl ein Maximum.

\section{V Coronae 1883}

Gemeint ist der Stern DM. $+40^{\circ} 2929$, dessen Ort nach Birmingham $1855^{\text {: }} \mathrm{I}^{\mathrm{h}} 44^{\mathrm{m}} 2 \mathrm{Is}+40^{\circ} \mathrm{I}$.

1883 Febr. bis Anfang Mai war der Stern am Sucher unsichtbar, Mai $9=10^{\mathrm{m} \cdot 7}$ am Refr. Juli 23, als $\mathrm{V}=99^{\mathrm{m}} \cdot \mathbf{2}$, begann ich die Beob. am Sucher. Das Max. ward gut bestimnt, da der Stern abnahm, lange bevor er in der nordwestlichen Dämmerung unsichtbar ward; im stärksten Lichte verharrte er gegen 20 Tage. $M$ ax. $188_{3}$ Sept. 5 , h vielleicht $=7^{\mathrm{m}} \cdot 7$.

$$
\text { 32. } x x^{\prime} \text { Coronae australis } 1883 \text {. }
$$

Die beiden oft unsichtbaren, dann, wenn sichtbar, lichtschwachen Sterne befinden sich an den Endpunkten eines sehr matten, bei J. Herschel fehlenden Nebels, der in $-37^{\circ}$ Decl, östlich in der Nähe von $h 377^{\circ}$ steht. Den Nebel und den westlichen Stern $x$ kannte ich schon 1861 ; $x^{\prime}$ und seine Veränderlichkeit fand ich erst seit 1876 . Nur $x$ kann $9^{\mathrm{m}} \cdot 5$ erreichen, aber der 5 f. Refr. ist fur Sterne der Art, die nur die Höhe von $15^{\circ}$ erreichen können, durchaus unzureichend, und viele Tage gehen allmonatlich wegen des Mondscheines verioren. Selten sieht man $x$ und $x^{\prime}$ gleichzeitig, und wohl eben so oft fehlen sie völlig, und man bemerkt nur den Nebel. Die Anwendung der grossen Telescope auf südlichen Sternwarten würde das Räthselhafte der Erscheinung längst gelöst und die Frage erledigt haben, ob $x$ und $x$ ' Sterne oder nur Verdichtungen im Nebel sind; ebenso wäre die Frage wegen der Periode so grosser Lichtänderungen leicht $2 \mathrm{u}$ beantworten. Die Athener Beobachtungen werden keine Entscheidung in Aussicht stellen können. Für x lässt sich, da er die ro. Grösse erreicht, ein $\mathrm{M}$ a x. bestimmen, nämlich 1883 Sept. $29.8, h=10^{\mathrm{m}}$. Die Periode habe ich ehemals zu 30 oder 31 Tagen angegeben.

\section{3. z Coronae australis 1883 .}

2 ward im October schwächer, als ich ihn jemals sah. Die unregelmässigen Aenderungen des Lichtes, die einst bestinmt eine Periode von 6 Tagen andeuteten, vermag ich jetzt nicht befriedigend durch Curven darzustellen. Hell war $z$ Ende Juni, dann Aug. 3, Aug. 30, schwach Juli 10, Juli 26, Juli 31, Aug. 8, Aug. 27, Sept. 28, Oct. 24. z steht nahe südlich von $x x^{\prime}$.

\section{4. $\chi$ Cygni 1883}

1882 Dec. 27 war $\chi=9^{\mathrm{m}} \cdot 7$. 1883 Mai $27 \chi=12^{\mathrm{m}} \cdot 2$ langsam zunehmend, mit merklicher Spur der Röthe. Als später der Stern dem freien Auge sichtbar ward, Sept. I 9 bis Nov. 19, erreichte er höchstens die Grösse 6.2. Er ward mit 2 Nachbarn am Sucher, mit einem der Nachbarn auch ohne Fernrohr verglichen. Zu- und Abnahme nahezu gleich. 3 Curven zeigen:

$$
\begin{aligned}
& \text { Max. } 1883 \text { Oct. } 16.5 \text { nach } b \quad p=I \\
& \text { - 15.5 } A=3 \\
& \text { - } 13.5 \text { fr. Auge }=2
\end{aligned}
$$

Mittel: $M$ ax. Oct. 1 5.0, $h=6^{\mathrm{m}} \mathbf{2}$. Die Abnahıne ward am Sucher noch bis zu Ende des Jahres beobachtet. - Letzte Periode $=408.5$ Tage.

\section{R Cygni I883.}

1883 Mai $7 \mathrm{R}=12^{\mathrm{m}} \cdot 3$. Juni 26 , Juli $\mathrm{I}_{3}$ unsichtbar am Refr. Aug. $26=12^{\mathrm{m}} \cdot 5$. Sept. 3 nicht sicher bemerkt. Sept. $6=12^{\mathrm{m}} \cdot 7$. Oct. $5=11^{\mathrm{m}} \cdot 6$. Von nun an ward $R$ oft an beiden Instrumenten mit 2 Nachbarsternen verglichen bis Mitte Januar 1884, als die Abnahme gewiss begonnen hatte. Nach 2 Curven setze ich:

Max. 1883 Dec. I 5, $\mathrm{h}=6 \mathrm{~m} .8$. Letzte Periode $=$ 428 'Tage.

$$
\text { 36. V Cygni } 1883 \text {. }
$$

Birmingham's rother Stern, Ort nach Schönfeld I 855 : $20^{\mathrm{h}} 15^{\mathrm{m}} 7^{\mathrm{s}}+47^{\circ} 26^{\prime} 3$. Aus den diesmal sehr zahlreichen Beobachtungen an beiden Instrumenten lassen sich die Curven nur befriedigend zeichnen, wenn man zugiebt, dass ein gewöhnliches einfaches Maximum nicht stattgefunden habe. Man ist genöthigt, 2 Maxima mit 2 wischenliegendem secun. dären Minimum anzunehmen. Der Einfluss des Mondlichtes auf die Helligkeit des tiefrothen Sternes ist für mein Auge nicht so stark, wie bei $\mathbf{R}$ Leonis und $\mathbf{R}$ Leporis. Der Stern

\begin{tabular}{|c|c|c|c|}
\hline I. Max. & sec. Min. & II. Max. & Bemerkungen \\
\hline Aug. I 6 & Sept. 29 & Nov. 12 & $p=3$ nach $a$ \\
\hline 9 & 23 & 7 & $=2, A$ \\
\hline 4 & I 3 & 2 & $=\mathbf{I}, B$ \\
\hline Aug. 12 & Sept. 24 & Nov. 9 & Mittel, bestimmt am Refractor. \\
\hline Aug. 18 & Sept. 25 & Nov. 6 & $p=3$ nach $a$ \\
\hline 16 & 28 & 12 & $=2, A$ \\
\hline I 3 & 25 & I 5 & $=\mathrm{I}, B$ \\
\hline
\end{tabular}
hat im Mai und Juni sehr rasch zugenommen, und sich von Anfang Juli bis Anfang December nur wenig verändert. Seit der Mitte des Dec. begann rasche Abnahme. Für 1883 hat man nach 3 Curven:

Aug. I 6 Sept. 26 Nov. 9 Mittel, bestimmt am Sucher.

Gebe ich in diesem Falle den Beob. am $R \in$ fr. das doppelte Gewicht, da ich den Stern am Sucher nur schwer 
sehe, so war I. Max. Aug. 1 3, se c. M i n. Sept. 25. II. M a x. Nov. 9 .

Der Abstand beider Maxima $=88$ 'Tage, das sec. Min. in der Mitte zwischen beiden. Max. d. Helle $=8^{m} \cdot 5$. Es war $V$ Juni $7=1_{1}^{m}$, Juni $26=9^{m} \cdot 7$, Juli $9=9^{m} \cdot 3$, Juli $22=8 \cdot 9$.

\section{Nova Cygni (von 1876) r883.}

Mai 27 , Juni $7,24,28$, Juli 3, 6, 25, 27, Aug: 7, 25 , Sept. 3, 8; Nov. 24, Dec. 23 ward der Ort des Sternes am Refr. sorgfältig untersucht, doch ward, 3 Nächte ausgenommen, keine Spur der Nova gesehen. Nur an den besonders guinstigen Abenden Juni 28, Juli 3, Aug. 25 ward an 3 Ocularen der Stern wirklich erkannt, etwa in der Helle $13^{\mathrm{m}} \cdot 5$, und der gezeichnete Ort in völliger Uebereinmit den Zeichnungen gefunden, die ich nach 1877 erhalten habe.

\section{R Camelopardali 1883.}

In Oct. 1882 begann am Sucher die Beob. für das zunehmende Licht, als $R=9 \cdot 3$; sie endete, als $x^{\mathrm{m}} 88_{3}$ Febr. I I der Stern am Sucher verschwand. Nach 2 Curven finde ich:

M a x. 1882 Dec, 28.5, $h=8^{\mathrm{m}} \cdot 5$. Erst Juni 9 sah ich ihn wieder $=9 \cdot 5$. Es erfolgte rasche Zunahme, langes Verweilen im Max. und seit Oct. I I rasche Abnahme, so dass er Nov. 19 unsichtbar ward. Nach 2 Curven setze ich: Max. 1883 Sept. 13. $h=8^{m}$. Periode $25^{8.5}$ Tage.

\section{Variab. Canis minoris (Haxendell) 1883 .}

Ort $1879: 7^{\mathrm{h}} 34^{\mathrm{m}} 4^{6^{\mathrm{s}}}+8^{\circ} 39^{\prime} 6$ nach Lohse, auch A. N. Nr. 2309 . Als der Stem im Nov. 1882 sehr schwach ward, konnte er am 5 f. Refr. mit einem sehr kleinen südlichen Nachbarstern verglichen werden bis März 1883. Aus I6 Beob. finde ich ziemlich sicher: Min. 1882 Dec. I4, $\mathrm{h}=12^{\mathrm{m}} \cdot 3$. Er ward ferner bis Juni 3 verfolgt, als er Abends in der Dämmerung unsichtbar ward. Als ich ihn Sept. 3 Morgens wiedersah, hatte die Abnahme seit dem letzten Max. (gegen Juli 22) schon begonnen. Ende Dec. 1883 war der Stern $12^{\mathrm{m}}$ oder schwächer.

\section{R Cassiopeae 1883 .}

Jan. 10 $R=8^{\mathrm{m}}$, Febr. $7=9^{\mathrm{m}}$, Aug. $6=9^{\mathrm{m}} \cdot 3$. Die Zunahme erfolgte rasch, die Abnahme sehr langsam; die Curven ergaben: Max. 1883 Nov. $21, h=6 \mathrm{~m} .6$. $R$ ward auch dem freien Auge sichtbar.

\section{S Cassiopeae 1883 .}

Im Aug. 1882 war $S$ am Sucher und auch am Refr. unsichtbar. Dec. 29 und $3 \mathrm{I} S=9^{10} 2$. Es ward aber erst I 883 März 6 der Ort genau untersucht, und es zeigte sich, dass das Max. schon vorüber war. März $5 \mathrm{~S}=8^{\mathrm{m}}$, April 3 $=8^{\mathrm{m}} \cdot 2$, April $27=9^{\mathrm{m}}$, Juni $28=9^{\mathrm{m}} \cdot 7$. Oct. 19 fand ich ihn unsichtbar am Refractor.

\section{S Cancri 1883 .}

Lichtschwach sah ich S 1883 März $12 \quad 7^{\text {h. }}$.2 und

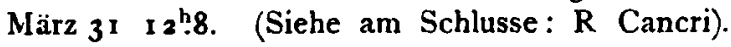

\section{S Delphini 1883 .}

Durch Herm Prof. Schönfeld daran erinnert, dass ich mich seither vergebens bemtiht hatte, den schwachen Stern (wie auch $\mathrm{T}$ Delphini) am Sucher zu beobachten, beeilte ich mich im Juni, durch Messungen die Oerter beider Sterne sicher zu ermitteln, und machte die Vergleichungen von nun an nur am Refr. Juli I I fand ich den richtigen Stern S. Er war $=9^{\mathrm{m}}$, nahm langsam $a b$, und war im Dec. $=12^{\mathrm{m}}$.

\section{T Delphini 1883 .}

Nach Ermittelung des richtigen Ortes ward der Stern von Juni 19 bis Ende Dec. am Refr. beobachtet. Die $\mathrm{Zu}$. nahme war sehr rasch, die Abnahme zuerst zögernd, seit Ende Oct. sehr rasch. Aus 2 Curven entnehme ich recht sicher: $\mathrm{M}$ ax. $\mathrm{I}^{88_{3}}$ Oct. $\mathrm{I}, \mathrm{h}=8^{\mathrm{m}} \cdot \mathbf{4}$.

\section{R Draconis 1883}

1882 Nov. 9 sah ich am Sucher die letzte Spur, und fand ihn unsichtbar bis 1883 März 31 , als $R=9^{m} \cdot 7$. Vollständige Beob. umfassen die Zeit von April 20 bis Anfang Juli. Die Curven ergaben ein gutes Resultat: Max. I883 Mai 8.5, h $=8^{\mathrm{m}} \cdot 7$. Zunahme sehr rasch, Abnahme lang. sam, zumal am Anfange; erst seit Juni 22 ward sie sehr beschleunigt. Juli $6 \mathrm{R}=9 ! 8$. Aug. 24 unsichtbar am Sucher. So blieb er bis Nov. a . Die regelmässigen, Nov. 28 begonnenen Beob. zeigten 1884 Jan. I 7 noch keine Abnahme.

\section{R Herculis 1883 .}

Mai $29 R$ am Sucher unsichtbar, Juni 26 am Refr. $=11 ! 7$, Juli 9, 24, 25 unsichtbar, Aug. 7 schwächer als 1 $2^{\mathrm{m}}$, Aug. 24 nicht sichtbar. Dann sah ich ihn zunehmend bis. Nov. 4 , so dass aber von Oct. 10 bis Nov. 4 keine Veränderung bemerkt ward; doch war die Lage zuletzt schon sehr ungünstig. Alle diese Angaben scheinen wenig sicher.

\section{S Herculis 1883 .}

März 31 bis April 30 war $S$ am Sucher unsichtbar. Mai I S am Refr. $=9 \cdot 8$, Mai $3 \mathrm{I}=10^{\prime \prime \prime} \cdot 7$. Juni 23 ward er am Sucher schwierig sichtbar. Aus Vergleichungen mit 2 Sternen ergab sich:

$\mathrm{M}$ ax. 1883 Sept. $12.8, \mathrm{~h}=7^{\mathrm{m}}$. Oct. 29 war $\mathrm{S}=9^{\mathrm{m}} \cdot 2$.

\section{T Herculis 1883 .}

April 15 bis Mai I $T$ am Sucher nicht kenntlich; Mai I fand ich ihn auch am Refr. nicht. Mai $7=12^{\mathrm{m}} \cdot 7$ Mit Juni 4 begann ich die Beob. am Sucher, als $T=9{ }^{\mathrm{m}} \cdot 4$ und beschloss sie Aug. 14 , als $T=9 \cdot 7$. Ziemlich sicher ergab sich:

Max. 1883 Juni $30, h=8$ : $_{2}$. Zunahme mässig rasch, Abnahme Juli 1-13 langsam, dann schneller. Für das Min. begann ich die Beob. mit dem Refr. Aug. 24. Der Stern war Sept. 30 bis Oct. $6=12^{m} \cdot 7$ und zweifelhaft zu erkennen; eine Curve giebt, wie ich glaube, genügend sicher die Zeit des kleinsten Lichtes: $M$ in. 1883 Sept. $29, h=12^{m} \cdot 7$. Das folgende Max. war nicht genau zu bestimmen, da sich der Stern dem N.W.-Horizonte zu sehr näherte. Dec. 1-15 fehlen wegen Mond und Wolken die Beob. Ich setze: Max. 1883 Dec. I 4, $h=7^{m} \cdot 5$. Periode $=167$ Tage. 


\section{U Herculis 1883 .}

1882 Oct. 29 bis Nov. 7 ward $U$ auch am Refr. nicht gesehen. Seit $188_{3}$ März $3 \mathrm{r}$ beob. ich den Stern am Sucher. Die Curve zeigt indessen, dass die Vergleichungen einen Monat früher hätten beginnen müssen. Ich erkenne nur, dass vielleicht April 5 das Max. eintrat, $h=7 \cdot 5$, wahrscheinlich aber früher. Juli $25 \mathrm{U}=8^{\mathrm{n}} \cdot 5$, Juli $30=9^{\mathrm{m}}$, Sept. $21=10^{\mathrm{m}}$, Oct. $19=11^{\mathrm{m}}$, Nor. $4=12^{\mathrm{m}}$. Dec. 22 sehr schwach.

\section{R Leonis majoris 1883 .}

Im März und April, als $\mathrm{R}$ grosse Helle hatte, war beide Male das Mondlicht sehr störend, so dass bei diesem sehr rothen Sterne die Lichtcurve 2 Maxima ergiebt, März 2 I und April 1 7. Wenn ich indessen auch die Beob. zur Zeit des Vollmondes nicht berücksichtige, bleibt doch eine starke Einbuchtung der Curve, welche zeigt:

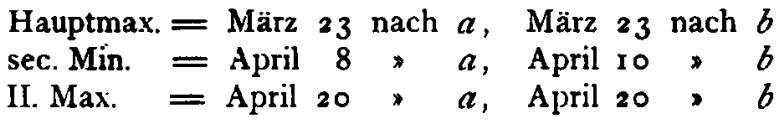

Die Abnahme erfolgte nach April zo zuerst sehr rasch, dann war sie im Mai sehr langsam. Juni $23 \mathrm{R}=8^{\mathrm{m}} \cdot 5$. Oct. 2 I-28 war $R$ sehr schwach, Nov. 24 war die Zunahme schon entschieden.

\section{5r. R Leonis minoris 1883 .}

1882 Dec. 2-13 $R$ am Sucher unsichtbar. Ebenso 1883 Febr. 2 bis Mai 7. Mai 9 vermuthete ich ihn $=9^{1 ! 1} 5$ und begann die Beob. Mai 24, als $R=99^{\mathrm{m}}$. Bis Ende Juli ward er viel am Sucher verglichen. Da aber der Stern seit der Mitte des Juli tief in N.W. in der Dämmerung stand, ist auf ein genaues Resultat nicht zu rechnen. Ich fand:

Max. 1883 Juni 29, h vielleicht $7^{\text {ul }}$. Oct. 2 I bis Dec. 22 war $R$ unsichtbar.

\section{R Leporis (crimson star) 1883 .}

I 882 Aug. 26 bis 1883 April ${ }_{5} 5$ zeigen zahlreiche Beob. an beiden Telescopen, dass der Stern langsam zunahm. Wie gewöhnlich, war der Einfluss der Dämmerung und des Mondlichtes auf den rothen Stern überaus gross und störend. Das Max. fiel auf die Zeit der allgem. Unsichtbarkeit des Sternes zur Zejt der Conjunction. Aus Beob. am Sucher ergab sich :

Min. ${ }^{188_{3}}$ Oct. I $_{7}$ Im Dec. hatte $\mathrm{R}$ bereits stark zugenommen.

\section{Var. Monocerotis 1883 .}

Es ist in h 399 ein schwacher Stern, oder eine Verdichtung an der Südecke eines Nebels, dessen Veränderlichkeit ich I86I erkannte. Die Periode zu bestimmen, ist bis jetzt nicht gelungen. 1883 Jan 2 bis April 29 erschien der Stern etwa $12^{\mathrm{m}}$, wohl langsam zunehmend. Sept. 3 war er unsichtbar oder $13^{\mathrm{m}}$, Oct. $7=12^{\mathrm{n}} \cdot 3$, Nov. 4 bei sehr guter Luft ganz unsichtbar, und auch der Nebel selbst so schwach, wie ich ihn seit 22 Jahren niemals gesehen zu haben glaube. Ueber die Existenz des Nebels konnte ich am 5f. Refr. nur mit Mühe Gewissheit erlangen. Ebenso fand ich ihn Nov. 23, und bis Ende des Jahres ward der Stern nicht sichtbar. Der Nebel konnte aber zuletzt doch etwas leichter gesehen werden, als im November.

\section{I" Orionis 1883 .}

Der sehr stark veränderliche Stern liegt im südlichen bogenförmigen Arme des grossen Orionnebels. Im Min. kann er für den $5 f$. Refr, verschwinden. Im Max. erreicit er $9{ }^{m} \cdot 5$. Für jetzt unterlasse ich noch die genauere Untersuchung. Sehr schwach war der Stern Jan. 25, 26, Nor. 23, 24, Dec. I 5, I 7 .

\section{R Pegasi 1883 .}

Jan. 2-28 nicht sichtbar am Sucher. Juli 29 neblige Spur. Aug. 25 am Refr. $=I^{\mathbf{m}}$. Bis Dec. 16 glaubte ich einigemal eine schwache Spur des Sternes am Sucher zu erkennen.

\section{T Pegasi 1883 .}

Von Jan. bis Iec. ward ' $\mathrm{I}$ in $3^{6}$ Beob. am Sucher niemals wahrgenommen. Aug. 26 am Refr. 'I' schwächer als I $^{\text {it }}$

\section{R Piscium 1883 .}

Am Anfange des Jan. war $R$ abnehmend, kaum noch 8.5. Ende Jan. $=10^{\text {nd }}$ am Refr. Juli a $R$ am Sucher nicht sichtbar. Sept. 6 glaubte ich ihn zu erkennen, bega a die Beob. aber erst Oct. 5, als $R=9^{\mathrm{m} \cdot 2}$. Häufige bis Mitte Jan. I 884 fortgesetzte Beob. haben nach 2 Curven ergeben:

Max. 1883 Nov. $17 \cdot 5, \quad h=7^{m} \cdot 5$.

\section{V Piscium 1883.}

In diesem Jahre ward $V$ ebenso wenig am Sucher gesehen, als in fruheren Jahren.

\section{R Persei 1883 .}

Seit 1882 Dec. 8 glaubte ich mehrmals die Spur von $\mathrm{R}$ am Sucher zu erkennen; Dec. 26 war er sicher $=9$ "' 3 . Die Zunahme war sehr rasch; das Mondlicht störte viele Tage lang. Ich finde, sehr wenig sicher, aus einer Curve:

Max. I883 Jan. 20, $h=8^{\mathrm{m}} 2$. Febr. I $\mathrm{R}=8^{\mathrm{m}} \cdot 5$, März 4 noch eine Spur, März 8 unsichtbar am Sucher. Juli $29 \mathrm{R}=9^{\mathrm{m}}$. Aus Beob. bis Mitte October folgt eine bessere Bestimmung des grössten Lichtes; ich fand: Max. I 883 Aug. 27.0, $h=8 ! 5$. Von Ende Oct. bis zu Ende des Jahres blieb $\mathbf{R}$ unsichtbar am Sucher.

\section{6o. R Ursae r883.}

1882 Juli 4 bis Dec. 9 war der Stern für den Sucher unsichtbar. Dec. $9 \mathrm{R}=9^{\mathrm{m}} \cdot 5$. Bis Dec. 26 erfolgte eine sehr rasche Zunahme; die Abnahme war langsamer. Ich finde aus der Curve:

Max. 1882 Dec. $3 \mathrm{r} \cdot 7, h=7^{\mathrm{m}} \cdot 7$. Es fehlen aber Beob. zwischen Dec. I 3 und 26 . Die Abnahme war so lang. sam, dass $188_{3}$ Febr. 3 der Stern nur 2 Stufen an Licht verloren hatte. März $26 \mathrm{R}=9^{\mathrm{m}}{ }^{2}$. April 26 unsichtbar. 
So blieb er unsichtbar am Sucher für lange Zeit. Oct. I9 fand ich $R=9^{\mathrm{m}}$. Früher, Aug. 24, fand ich ihn am Refr. $=11^{\mathrm{m}}$. Sept. $5=10^{\mathrm{m}} 7$. Sept. 30 am Orte von $R 2$ Sterne $1 \mathrm{x}^{\mathrm{m}}$, Oct. $6 \mathrm{R}=10^{\mathrm{m}}$. Die späteren Beob. haben ergeben: Max. 1883 Nov. I $_{3}, h=7^{\mathrm{m}} \cdot \mathbf{7}$. Die $\mathrm{Zu}$ nahme geschah viel rascher als die Abnahme.

\section{S Ursae 1883 .}

Jan. ro $S=9^{\prime \prime \prime} 7$, dann unsichtbar für den Sucher bis April 11. Am 24, April $S=9$ m. Max. 1883 Juni 14, $\mathbf{h}=7^{\mathrm{m}} \cdot 7$. Zu- und Abnahme nahezu gleichförmig. Sept. 5 $\mathrm{S}=\mathrm{I}^{\mathrm{m}}$ am Refr., Oct. $6=\mathrm{II}^{\mathrm{m}} 7$, Dec. $4=9^{\mathrm{m}}$.

\section{T Ursae 1883 .}

1882 Oct. 19 bis Dec. 3 I $\mathrm{T}$ unsichtbar am Sucher, ebenso 1883 Jan. 12 bis März 8. Am 26. März $T=8{ }^{m} 5$. Max. 1883 April 20, $h=7 \% 6$. Die Zunahme sehr rasch, die Abnahme sehr langsam. Juli 25 war ${ }^{\circ} T=9^{\mathrm{m}} \cdot 5$, Aug. 5 $=9 \cdot 7$, Sept. 4 unsichtbar. Er blieb so bis Nov. 24; Dec. $6=9^{\mathrm{m}}$, Dec. $20=7^{\mathrm{m}} \cdot 2$.

\section{R Tauri 1883 .}

1882 Nov. 27 ward $R$ für den Sucher unsichtbar, und ward bis zum nächsten April nicht gesehen. Juli 29 und Aug. 3 unsichtbar, Aug. ro $=9^{\text {m. }}$ Aus vielen Beob. bis Ende October finde ich:

Max. $188_{3}$ Sept. 15, h= 8:m. Nov. I $9 R=9^{m} \cdot 5$. Dec. I6 $R$ unsichtbar am Sucher.

\section{R Sagittae 1883 .}

Mai 27 bis Juni 4 fast constant hell, und ähnlich bis in den Juli, bis er seit Juli 13 abzunehmen anfing. In jedem Monate müssen die Beob. eine Woche lang wegen des Mondes ausgesetzt werden. Ich finde :

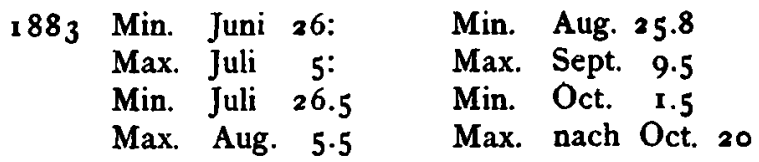

Zunahme rascher als Abnahme.

\section{U Sagittarii 1883.}

Alle Beob. am Sucher. Im Mai und Nov. ist der Stern Abends dem Horizonte nahe. In jedem Monate eine 2- bis 3 tägige Störung durch den Mond (Nr. 65, 66, 67 sind nun I 8 Jahre lang beobachtet).

\begin{tabular}{|c|c|c|c|c|c|}
\hline \multicolumn{3}{|c|}{ Maxima. } & \multicolumn{3}{|c|}{ Minima. } \\
\hline Mai & $29.00 p=$ & 3 & Juni & $1.40 p=$ & 2 \\
\hline Juni & 4.75 & 3 & & 8.85 & 3 \\
\hline & 10.82 & 2 & & 15.00 & 3 \\
\hline & 17.75 & 3 & & 21.60 & 0.5 \\
\hline & 23.87 & 3 & & 28.25 & 3 \\
\hline uli & 1.50 & 4 & Juli & 5.20 & 3 \\
\hline & 7.75 & 3 & & $11.4^{\circ}$ & 4 \\
\hline & 14.70 & 4 & & 18.40 & 4 \\
\hline & 22.00 & 4 & & 25.75 & 4 \\
\hline
\end{tabular}

8d. 108.

\begin{tabular}{|c|c|c|c|c|c|c|}
\hline \multicolumn{3}{|c|}{ Maxima } & \multicolumn{4}{|c|}{ Minima. } \\
\hline Juli & $29.33 p=$ & 3 & Aug. & 1.37 & $p=$ & 4 \\
\hline Aug. & 4.20 & 4 & & 7.95 & & 4 \\
\hline & 10.75 & 3 & & 14.00 & & 0.5 \\
\hline & 16.60 & 0.5 & & 21.12 & & $\mathbf{1}$ \\
\hline & 23.82 & 2 & & 27.90 & & 4 \\
\hline & 30.85 & 4 & Sept. & $3 \cdot 33$ & & 4 \\
\hline Sept. & 6.87 & 4 & & 10.70 & & 3 \\
\hline & 13.80 & 3 & & 17.42 & & 4 \\
\hline & 20.15 & 2 & & 24.35 & & 3 \\
\hline & 27.77 & $\mathbf{r}$ & Oct. & 1.10 & & 4 \\
\hline Oct. & 4.65 & $\mathbf{I}$ & & 7.75 & & 0.5 \\
\hline & 11.80 & 0.5 & & 14.77 & & 0 \\
\hline & 17.07 & 2 & & 20.62 & & 4 \\
\hline & 23.80 & 3 & Nor. & 4.25 & & 2 \\
\hline & 30.70 & $\mathbf{I}$ & & 11.25 & & $\mathbf{I}$ \\
\hline Nov. & 7.60 & 0.5 & & 18.00 & & 0.5 \\
\hline & I 3.75 & 2 & & & & \\
\hline
\end{tabular}

66. 3 Fl. $=$ X Sagittarii 1883 .

Die Beob. geschahen nur mit freiem Auge. In jedem Monate erleiden die Beob. starke Unterbrechung durch den Mond. Im Mai und September-October ist der Stern Abends dem Horizont zu nahe.

Maxima.

$\begin{array}{ccc}\text { Mai } & 31.90 & p=2 \\ \text { Juni } & 6.55 & 2 \\ 13.55 & 2 \\ & 27.95 & 4 \\ \text { Juli } & 5.37 & 3 \\ 12.00 & 4 \\ & 25.75 & 4 \\ \text { Aug. } \quad 1.70 & 4 \\ & 8.90 & 4 \\ & 22.40 & 0.5 \\ 30.05 & 3 \\ \text { Sept. } & 6.50 & 2 \\ 20.10 & 0.5 \\ 27.50 & 0.5 \\ & \end{array}$

\section{7. $\gamma^{1}=$ Wagittarii 1883}

Alle Vergleichungen am Sucher; die Beob. wurden weniger durch den Mond gestört, als die der beider vorigen Sterne. Im Mai und Oct. ist der Stern Abends den Horizont zu nahe.

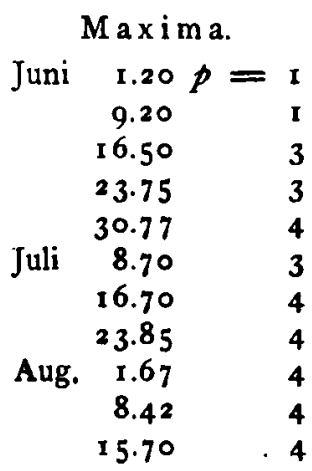

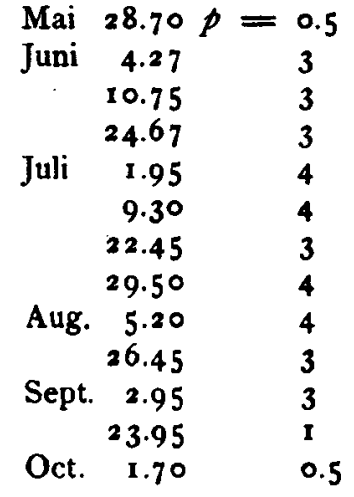

Minima.

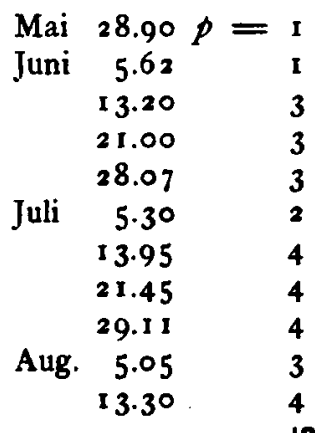


Maxima.

Aug. $23.87 p=4$ 30.95

Sept. 7.80 15.20

22.75

30.50

Oct. $\quad 7.75$

Nov. 7.75

\begin{tabular}{rc}
\multicolumn{3}{c}{ Minima. } \\
Aug. $20.75 p=3$ \\
27.55 & 4 \\
Sept. 5.25 & 4 \\
12.62 & 4 \\
20.75 & 3 \\
Oct. 5.3 & 0.5 \\
18.3 & 0.5 \\
Nov. 3.8 & 0.5 \\
11.0 & 0.5
\end{tabular}

Die Periode ist wenig regelmässig. Während der langsamen Abnahme zeigt sich eine Anomalie der Curve, ganz ähnlich der von $\eta$ Aquilae.

\section{R Scorpii 1883 .}

März 31, April 26, Mai I bis Juni 7 war $R$ ain Refr. unsichtbar. Juni $9=12^{\mathrm{m}}$. 2 Curven ergaben:

Max. 1883 Juli 11.5, $h=10{ }^{m} 3$. Juli $12-23$ war das Mondlicht sehr störend. Sept. $4 R=12 ! 8$ und später bis Ende October unsichtbar.

\section{S Scorpii 1883 .}

März 31, April 26 S am Refr. unsichtbar, Mai I = $1 \mathrm{I}^{\mathrm{m}}$. Nach Vergleichungen mit einem Sterne hat man: M ax. 1883 Mai 14.5, $h=10^{\mathrm{m}} .5$. Es war aber die Störung durch den Mond Mai I I-23 so bedeutend, dass über scheinbare Anomalien der Curve keine Entscheidung möglich ist. S nahm zuerst sehr langsam ab und schien Ende Mai ein wenig heller zu werden. Seit Juni 4 erfolgte rascheste Abnahme.

\section{Variab. Scorpii (Palisa) I883.}

Ort $1877: \quad 16^{\mathrm{h}} 4^{\mathrm{m}} 35^{\mathrm{s}}-19^{\circ}{ }_{48}: 9$. Zu keiner Zeit habe ich den Stern in diesem Jahre sicher gesehen. Als Spur glaubte ich ihn zu erkennen: Juli 7, Aug. 28, Sept. I, jedesmal am Refr.

\section{R Scuti 1883 .}

Die Vergleichungen mit 3 Nachbarsternen umfassen die Zeit von Mai 9 bis Dec. 17. Bis Aug. 20 zeigte sich allgemeine langsame Abnahme, mit sec. Max. und Min. Juni 15 und Juli 18 . Dann erfolgte seit Aug. 19 eine merkwürdige und so rasche Abnahme, wie ich sie seit vielen
Jahren nicht gesehen habe. Am 13. Sept. erreichte der Stern ein ausgezeichnetes, lichtschwaches $\mathrm{Minimum}$, in welchem er wohl nur die 9. Grösse hatte. Von Sept. 13 bis Oct. 6 nahm er ungemein schnell an Helligkeit zu, und erreichte bald sein gewöhnliches Licht, nahe $6^{\mathrm{m}}$. So blieb er bis Mitte Dec., als er westlich in der Dämmerung verschwand. Dec. $22 \mathrm{um} I 7^{\mathrm{h}} \cdot 5$ war er noch hell, doch geringer als $6^{\mathbf{m}}$.

\section{R Serpentis 1883 .}

Seit Oct. $188_{2}$ war $R$ am Sucher unsichtbar. $188_{3}$ Febr. $3 \mathrm{R}=7^{\mathrm{m}} \cdot 5, \quad$ Febr. $28=8^{\mathrm{m}}, \quad$ März $7=8^{\mathrm{m}} \cdot \mathrm{7}$ April $3=9^{\mathrm{m}} \cdot 7$. Die Beob. des kleinsten Lichtes misslang. Oct. $2 \mathrm{R}=\mathrm{II}_{\mathrm{m}}^{\mathrm{m}}, \quad$ Oct. $\mathrm{r} 9=10^{\mathrm{m}}, \quad$ Nov. $4=9^{\mathrm{m}} \cdot \mathrm{s}$, Dec. $22=7^{\mathrm{m}}$.

\section{R Virginis 1883 .}

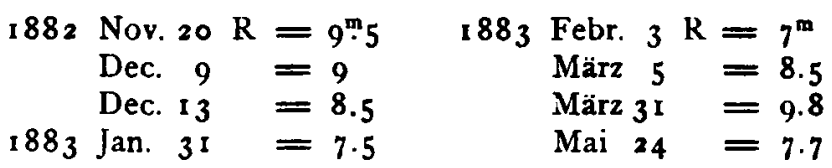

Die Beob. im Frühling sind zu wenig zahlreich; doch giebt eine Curve zu erkennen, dass gegen April is ein Minimum eingetreten sei. Das folgende Max. beob. ich am Sucher, und fand nach 2 Curven:

Max. 1883 Juni 2 r.o, $h=7$. $^{\mathrm{m}}$. Zunahme sehr rasch. Abnahme Juni 22 bis Juli 16 langsam, dann sehr rasch. Juli $31 \mathrm{R}=9{ }^{\mathrm{m}} 3$, Aug. $5=9{ }^{\mathrm{m}} \cdot 5$, Dec. $22=9 \cdot 5$.

\section{U Virginis 1883 .}

Jan. 3 I bis März 6 ward $U$ am Sucher nicht gesehen. Jan. $28 U=9^{m}$. Aus Beob. bis Mitte Aug. finde ich:

Max. 1883 Juni 18.5, $h=7.8$. Der Vollmond hat sehr gestört, rasche Zunahme, langsame Abnahme.

\section{R Cancri 1883 . (Nachträglich).}

Jan. $5 \quad R=9^{m}$, Jan. $25=9^{m} \cdot 3$, Febr. $12=10^{m}$, April $3 \mathrm{ro}^{\mathrm{m}}$ ?, Mai 4 nicht gesehen, Sept. $3=8^{\mathrm{m}} \cdot 5$, Sept. $3{ }^{\circ}$ $=7^{\mathrm{m}}$, Oct. $19=6^{\mathrm{m}} \cdot 5$, Oct. $28=6^{\mathrm{m}} \cdot 7$, Dec. $6=7^{\mathrm{m}} \cdot 7$, Dec. $20=8{ }^{\mathrm{m}} \mathrm{2}$, Dec. $3 \circ=8^{\mathrm{m}} \cdot 5$. Von Sept. 3 bis Dec. $3^{\circ}$ ward $R$ I 7 mal verglichen.

Max. $188_{3}$ Oct. $23, h=6{ }^{m} \cdot 5$, rasche Zunahme, etwas langsamere Abnahme. Mondlicht Oct. I9-2 I sehr störend. Letzte Periode $=3_{3} 3$ Tage.

Athen 1884 Jan. 22.

F. F. Ful. Schmidt.

\section{Proper Motion of $\mathrm{AOe}_{2} 22545$.}

This star has a proper motion of nearly $\mathbf{I}$. The proper motion in Dec. is almost insensible; in RA. it is approximately - $0.07 \mathrm{x}$. It has been observed by Argelander ( $\mathrm{AOe}_{2} 22545$ ), Schmidt (BB. VI, pag. 365 No.62), in the Washington Zones (Mur. 146, Trans. 143), Yarnall (10114) and Stone (1 r973). 